\title{
Genetic Counselling for the Laboratory: building bridges between disciplines
}

\author{
Domenico Coviello $\mathbb{D}^{1} \cdot$ Virginia Bizzarri ${ }^{2} \cdot$ Kevin McGhee $^{3} \cdot$ Ramona Moldovan $^{4}$
}

Received: 7 September 2018 / Accepted: 7 September 2018 / Published online: 22 October 2018

(c) European Society of Human Genetics 2018

Practical Genetics Counseling for the laboratory

Edited by: McKinsey L. Goodenberger, Brittany C. Thomas \& Teresa Kruisselbrink

Published by: Oxford University Press,

Year: 2017

ISBN: 9780190604929

Price: $£ 60.00 / \$ 60.00$

The Medical Genetics laboratory was once a sanctuary for Mendelian diseases and congenital malformation syndromes. Now however, we find ourselves in the era of medical genomics with a modern clinical service branching out into all fields of medicine. By utilising the technological advances made e.g. in next generation sequencing, we are no longer dealing solely with single genes disorders, but with a plethora of gene variants that contribute to the determination of phenotypes or predisposition to disease.

Today, DNA analysis generates terabytes of data, requiring significant contribution from Bioinformaticians. Putative DNA variants need to both be investigated in large numbers of subjects and to be validated using in vitro or in vivo functional studies. Specific variant databases need to be used in conjunction with "big data" from clinical databases. Thus, corroborating, translating and sharing clinical

Domenico Coviello

domenico.coviello@galliera.it

1 Laboratory of Human Genetics, Department of Genetic Sciences and I.B.M.D.R., E.O. Ospedali Galliera, Genova, Italy

2 Membro AIPA (Associazione Italiana Psicologia Analitica), Membro IAAP (International Association Analytical Psychology), Genova, Italy

3 Faculty of Science and Technology, Bournemouth University, Poole, UK

4 Department of Psychology, Faculty of Psychology and Educational Sciences, Babeș-Bolyai University, Cluj Napoca, Romania and molecular data are probably the most important challenges we face today.

Taking each one of these issues in turn, a modern genetics service must now become a genomics service that facilitates a multi-disciplinary approach. It must be economically viable using automation systems for DNA extraction and sequencing, reducing the need for technicians; consolidating smaller laboratories into fewer but larger laboratories, thereby optimizing the number of samples processed.

The enormous amount of data produced will require a larger number of bioinformaticians and clinical geneticists for the interpretation of results. Yet what "Practical Genetic Counseling for the Laboratory" shows us is that the role of the genetic counsellor is fundamental to the success of genomics services.

Genetic counselling was always an integrative part of genetic services because it is essential in helping people understand and adapt to the medical, psychological and familial implications of genetic contributions to disease (NSGC, 2006). Over the last five decades the genetic counselling profession has expanded globally to nearly 7000 genetic counsellors in over 28 countries [1]. A number of professional organisations (e.g. The European Board of Medical Genetics, The American Board of Genetic Counseling, The Australasian Board for Genetic Counseling, or the Canadian Association of Genetic Counselors Certification Board) advocate and regulate Genetic Counselling as a profession. The core competencies of genetic counsellors, the scope of practice, the training programmes, the mechanisms of credentialing share many similarities across the globe. Adding value to the care of patients with genetic conditions and their families is undoubtedly the overarching goal for all professionals in this area

"Practical Genetic Counseling for the Laboratory" approaches theoretical aspects relevant to genetic counselling and provides valuable practical information to genetic counsellors working both in laboratories and clinical settings. The authors skilfully discuss the key 
role genetic counsellors have both in direct contact with patients and families, and with colleagues involved at various stages of the patient's journey. In addition to discussing current roles and present issues facing genetic counsellors, the book also anticipates future developments and challenges for the genetic counselling profession, such as counselling supervision of other clinicians.

With contributions from scientists, geneticists and genetic counsellors, this book is meant to be a comprehensive textbook for the profession in how to translate, as well as effectively and meaningfully communicate genomic information to patients and their families. It explores the role of the genetic counsellor as the key to sharing genomic information between clinicians, laboratory technologists, patients and family members. It serves as a primer for the future role of genetic counsellors in genomic medicine.

\section{References}

1. Ormond KE, Laurino MY, Barlow-Stewart K, Wessels TM, Macaulay S, Austin J, Middleton A. Genetic counseling globally: Where are we now? Am J Med Genet 2018; 1; 98-107 